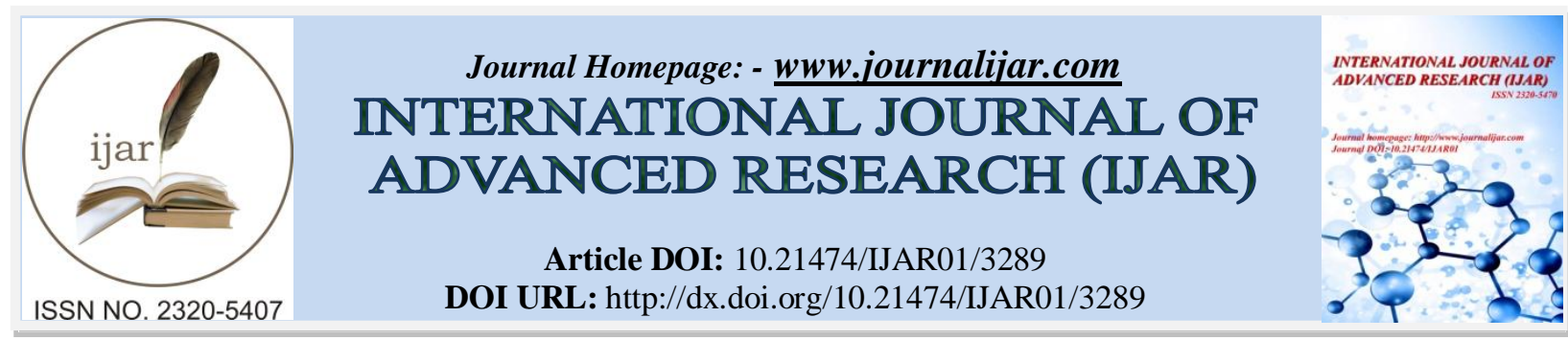

RESEARCH ARTICLE

\title{
RARE PRESENTATION OF DUODENAL ADENOCARCINOMA IN A YOUNG ADULT - A CASE REPORT.
}

\section{Dr. Midhun. P. Gopalakrishnan MS. DNB ${ }^{1}$, Dr. Satheesan B MS. DNB. MCh ${ }^{2}$, Dr. Dipin Jayaprakash $\mathrm{MS}^{1}$ and Dr. Basavaraj Ankalakotti MS.DNB ${ }^{3}$}

1. Senior Resident in Surgical Oncology, Malabar Cancer Centre, Thalassery, Kerala, India.

2. Director, Malabar Cancer Centre, Thalassery, Kerala, India.

3. Assistant Professor in Surgical Oncology, Malabar Cancer Centre, Thalassery, Kerala, India.

\section{Manuscript Info}

\section{Manuscript History}

Received: 10 December 2016

Final Accepted: 14 January 2017

Published: February 2017

Key words:-

Duodenum, Adenocarcinoma, Young adult

\section{Abstract}

The incidence of duodenal adenocarcinoma in general population is very rare. It may arise from duodenal polyps seen in familial polyposis or can be associated with celiac disease. Usually duodenal adenocarcinoma is seen in middle or elderly age group. It can occur in young age also when it is associated with polyposis syndromes or with any inflammatory diseases affecting small bowel. But when there is no such syndromes or diseases, duodenal adenocarcinoma in young age is very rare. We are reporting a case of 28 years old male who presented to us with history of recurrent abdominal pain. After evaluation it was diagnosed as adenocarcinoma arising from junction of first and second part of duodenum without any associated polyposis syndromes or inflammatory bowel diseases.

Copy Right, IJAR, 2017,. All rights reserved.

\section{Introduction:-}

Duodenal adenocarcinoma is an aggressive malignancy though it is seen rarely ${ }^{1}$. Primary malignant tumors of the duodenum makes $0.3 \%$ of all gastrointestinal tract tumors and up to $50 \%$ of small bowel malignancies. Duodenal adenocarcinoma must be differentiated from malignant tumors of the ampulla, pancreas and common bile duct ${ }^{2}$. It may arise from duodenal polyps seen in familial polyposis or can be associated with celiac disease. The common location of the tumour is second part of duodenum. Usually duodenal adenocarcinoma is seen in middle or elderly age group. It can occur in young age also when it is associated with polyposis syndromes or with any inflammatory diseases affecting small bowel. But when there is no such syndromes or diseases, duodenal adenocarcinoma in young age is very rare. We are reporting a case of 28 years old male who presented to us with history of recurrent abdominal pain. After evaluation it was diagnosed as adenocarcinoma arising from junction of first and second part of duodenum without any associated polyposis syndromes or inflammatory bowel diseases.

\section{Case Report:-}

28 years old male ECOG performance status 1 with no co morbidities presented to us with upper abdominal pain and vomiting of one month duration. There was no history of any malignancy in family. Local and systemic examination was within normal limits. Routine blood investigations were normal. Upper gastrointestinal scopy done showed (Fig 1 \& 2) ulcerating lesion present in D1 and D2 junction of 
duodenum with stenosis of that area. Endoscopic biopsy was suggestive of moderately differentiated adenocarcinoma of duodenum. Colonoscopy done was normal. Contrast enhanced CT scan showed (Fig 3) mild circumferential irregular wall thickening up to $1.3 \mathrm{~cm}$ in D1 part of duodenum with extension in to the D2 segment. There was a large node seen in the peripancreatic location superior and medial to the pancreatic head measuring $3.2 \mathrm{~cm}$ in size. Another node of size $2.8 \mathrm{~cm}$ present in the porta hepatis. No evidence of periduodenal fat stranding, para aortic or distal adenopathy or distant metastasis.

After completing evaluation Whipple's resection was done for this patient. Post operative period was uneventful. Histopathological report came as moderately differentiated adenocarcinoma duodenum with a tumour of size $3 * 3 \mathrm{cms}$ with $1.7 \mathrm{cms}$ thickness, infiltrating through the muscularis propria in to the sub serosal connective tissue and invades adjacent pancreatic tissue with positive lymphovascular invasion. Metastatic carcinomatous deposits in four out of twenty two lymph nodes dissected seen. Extra capsular spread in one lymph node noted. Periportal lymph node dissected is negative for malignancy. All resected surgical margins are free of malignancy. pT4b pN2 (AJCC $7^{\text {th }}$ edition).

After discussing the case in institutional multi specialty board, it was decided to give 8 cycles of chemotherapy (CAPEOX). Now the patient has completed 5 cycles of chemotherapy and is doing well.

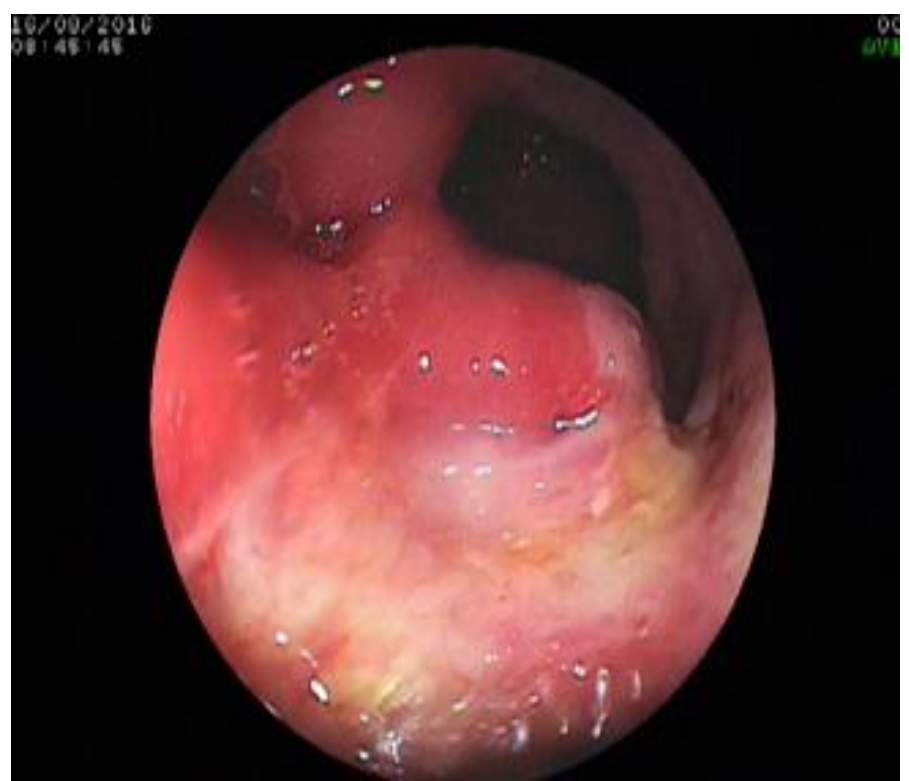

Fig 1:- Ulceroproliferative growth in D1 - D2 junction. 


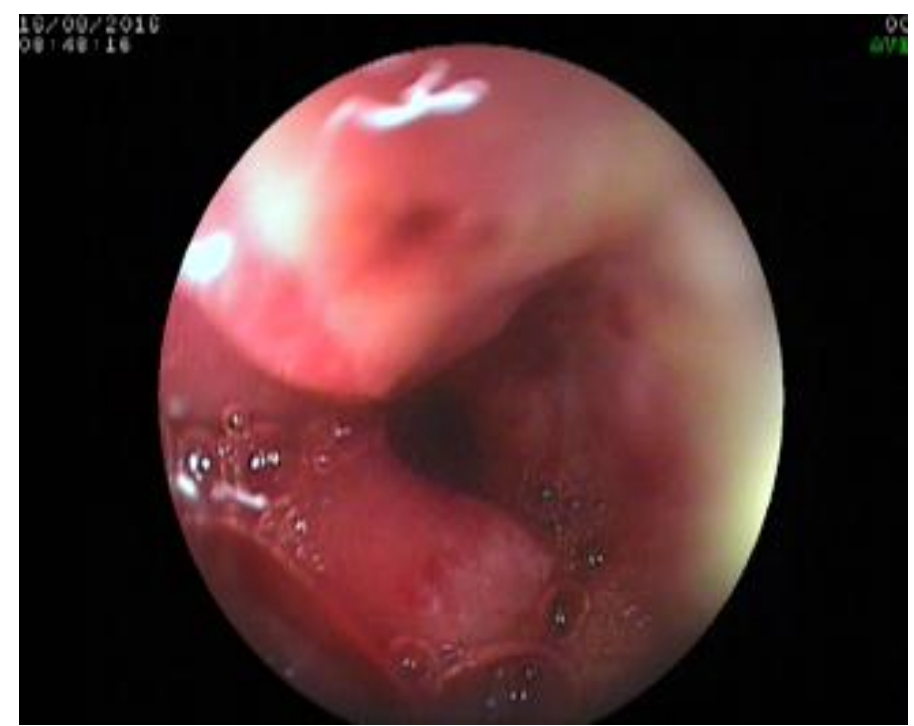

Fig 2:- Duodenal lesion causing D1 - D2 junction Stenosis

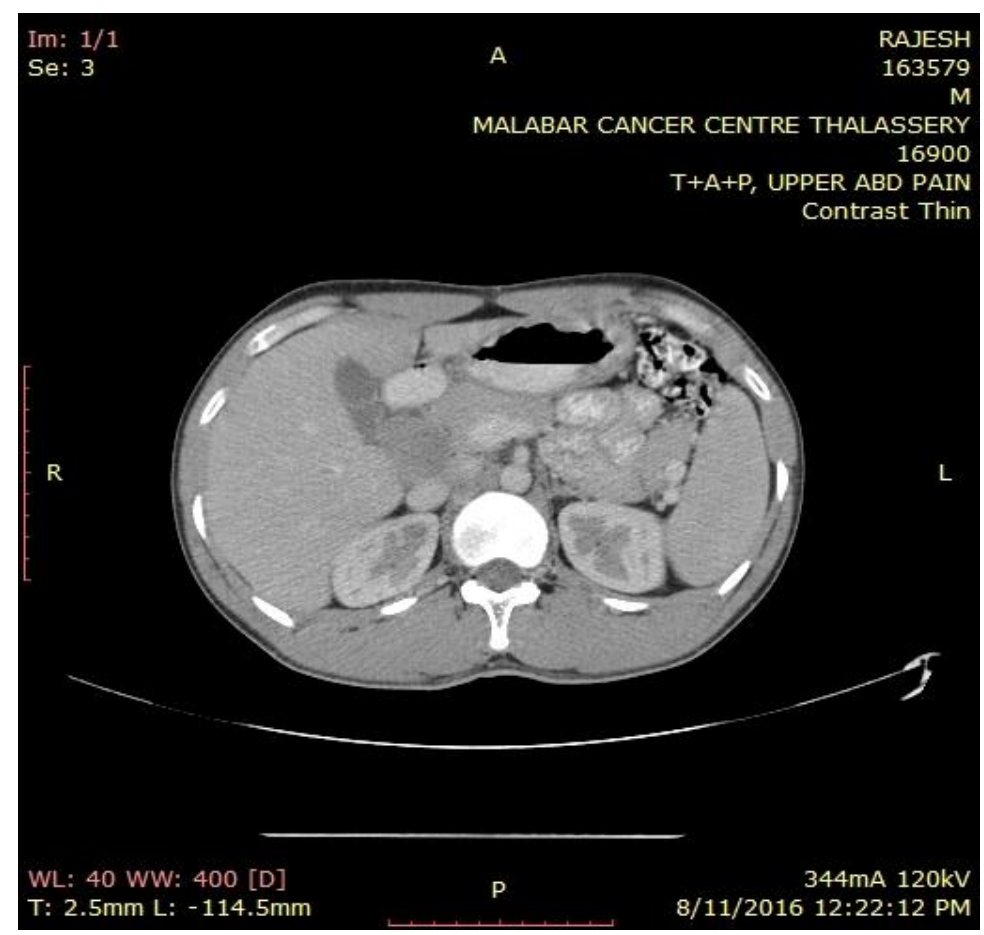

Fig 3:- CECT scan showing duodenal lesion with peripancreatic and periportal lymph nodes

\section{Discussion:-}

The most common tumour of the duodenum is adenocarcinoma ${ }^{3}$. The other histological varieties seen are carcinoid tumors, lymphomas, stromal tumours, leiomyosarcomas and gastrinomas. Adenocarcinoma of the duodenum may arise from duodenal polyps observed in familial adenomatous polyposis, Gardener's syndrome, Lynch syndrome, Muir Torre syndrome, Puetz Jeghers syndrome, and are or it can be associated with Crohn's disease and Celiac disease ${ }^{4}$. Duodenal adenocarcinoma is usually seen in middle or elderly age group. But it is seen in young age also when it is associated with polyposis syndromes or inflammatory diseases. But occurrence of duodenal adenocarcinoma without such syndromes in young age is very rare. 
The common symptoms of duodenal carcinoma is nausea and vomiting, mass in the abdomen, abdominal pain, acid reflux, unexplained weight loss, upper gastrointestinal bleeding and obstructive jaundice. The diagnostic investigations for duodenal carcinoma includes upper gastro intestinal endoscopy, contrast enhanced CT scan, barium study and routine blood investigations. Upper gastrointestinal endoscopy shows the exact location of the tumor and endoscopic biopsies can be taken to confirm the diagnosis. Contrast enhanced CT scan helps to confirm the anatomical location of the lesion, operability of the lesion and to rule out any metastasis. Barium study usually reveals an irregular stricture of the duodenum but it can be either benign or malignant stricture. If you are suspecting any syndromes like familial adenomatous polyposis colonoscopy should be done to rule out colonic polyps.

If the lesion is operable and non metastatic after evaluation the treatment is curative resection by Whipple's procedure. The 5 year survival rate varies widely according to different series published, but is generally reported to be $>40 \%$ in case of curative resection ${ }^{5}$. The presence of lymph node metastases is not a bad prognostic factor in most series and is not a contraindication for curative resection ${ }^{6}$.

\section{Conclusion:-}

Though rare, duodenal adenocarcinoma can occur in young age also. Primary malignant tumours of the duodenum must be differentiated from malignant tumours of the ampulla, pancreas and common bile duct. The prognosis of duodenal adenocarcinoma is better than peri ampullary, pancreatic or cholangiocarcinoma. Treatment of this condition is by curative resection.

\section{Conflict of Interest:-}

None

\section{Acknowledgements:-}

We sincerely appreciate the help, assistance and support offered by the faculty and staff members of Department of Surgical Oncology, Malabar Cancer Centre, Thalassery.

\section{References:-}

1. Jordan M Cloyd, Elizebeth George and Brenden C Visser. (2016): Duodenal adenocarcinoma : Advances in diagnosis and surgical management. World J Gastrointest Surg., 8(3) : 212-221.

2. Pierre - Louis Fagniez and Nelly Rotman. Malignant tumors of the duodenum. Holzheimer R G, Mannick J A, editors. Surgical Treatment : Evidence - Based and Problem - Oriented. Munich : Zuckschwerdt; 2001.

3. Cunningham J D, Aleali R, Aleali M, Brower S T and Aufses A H. (1997): Malignant bowel neoplasms. Histopathologic determinants of recurrence and survival. Ann Surg., 225 : 300-306.

4. Burt R W, Berenson M M, Lee R G and Tolman J W et al. (1984): Upper gastrointestinal polyps in Gardner's syndrome. Gastroenterology., $86: 295-301$.

5. Rotman N, Pezet D, Fagniez P L, Cherqui D, Celicout B and Lointier P. (1994): Adenocarcinoma of the duodenum: factors influencing survival. French Association for Surgical Research. Br J Surg., 81 : $83-85$.

6. Ohigashi H, Ishikawa O, Tamura $\mathrm{S}$ and Imaoka $\mathrm{S}$ et al. (1998): Pancreatic invasion as the prognostic indicator of duodenal adenocarcinoma treated by pancreatoduodenectomy plus extended lymphadenectomy. Surgery., $124: 510-515$. 